\title{
Approximating Method and Experimental Determinating Coefficients for an Electric-magnetic Drive System Model
}

\author{
Yan Zhang ${ }^{1}$, Xiangyu Cheng ${ }^{2,3,4, *}$ and Wenge Chen ${ }^{2}$ \\ ${ }^{1}$ Anhui Water Conservancy Technical College, Hefei 231603, China \\ ${ }^{2}$ High Magnetic Field Laboratory, Chinese Academy of sciences, Hefei 230031, China \\ ${ }^{3}$ University of Science and Technology of China, Hefei 230026, China \\ ${ }^{4}$ The 38th research institute of China Electronics Technology Group Corporation, Hefei 230088, China \\ ${ }^{*}$ Corresponding author
}

\begin{abstract}
We've developed a newer method to calculate the repulsive force between current-carrying coil and nearby metal plate. But it feels the final solution formula is complex. There are some unknown coefficients have not measured and an integral in the formula seems difficult to deal with. In the paper, we determinate these coefficients by experimental method firstly, and then propose solutions for some typical metal plate reflectors combining with Matlab procedure. Lastly, we verify the correctness of the factors and cases of classic metal plate reflectors in practice. Therefore we have solved the AC currentcarrying coil of electric-magnetic drive problem completely in the article.
\end{abstract}

Keywords-AC current-carrying coil; determinating coefficient; integral calculation; electric-magnetic drive

\section{INTRODUCTION}

In our former researches, we deduced a kind of method only in theory to calculate the repulsive force problem for AC current-carrying coil and its nearby metal plate system. We can't figure out the final numerical result because of the coefficient is unknown and a complicated integral existing in it seems hard to deal with. So we devote to find out the final solution thoroughly by certain approximating [1] and experimental method [2] within following parts.

\section{SIMPLIFICATION FORMULA FORM}

The formula [3] we got in our former researches

$$
\begin{aligned}
F_{\text {total }}= & -K_{T} \cdot \frac{K}{\sqrt{1+K^{2}}} \cdot L^{2} I^{2} \cdot \int \frac{d_{S}}{\mu d^{4}} \\
& \cdot \sin \left(\frac{4 \pi f d}{C_{\text {light }}}+\arcsin \frac{K}{\sqrt{1+K^{2}}}\right)
\end{aligned}
$$

is accurate in calculating force between a coil and induction plate, but still very complicated.

So firstly, we want to further simplify the formula in normal conditions: the distance $d$ between a coil and induction plate is often small, and the supply frequency $f$ is also normally not very large. These lead to a small value of $\frac{4 \pi f d}{C_{\text {light }}}$. So we can simplify calculate $F_{\text {total }}$ as follows:

$$
F_{\text {total }}=-K_{T} \cdot \frac{K^{2}}{1+K^{2}} \cdot L^{2} I^{2} \cdot \int \frac{d_{S}}{\mu d^{4}}
$$

where $K=k_{\mathrm{fB} \rho} \frac{f L I}{\rho d_{m}}=k_{\lambda B \rho} \frac{L I}{\lambda \rho d_{m}}, d_{m}$ is the distance from a coil to a induction plate under the coil.

For more closer approach to theoretical value, we set $d_{m}=\sqrt{d_{\min } \cdot d_{\max }}$ where $d_{\text {min }}$ is the minimum distance, $d_{\max }$ is the maximum distance between a coil and induction plate.

\section{EXPERIENCE FACTORS AND INTEGRAL CALCULATIONS}

\section{A. Experience Factors}

Nextly, we've measured its experience factors as follows:

$$
\left\{\begin{array}{l}
K_{T}=4.28 \times 10^{-9} \\
k_{f B \rho}=1.88 \times 10^{-11} \\
k_{\lambda B \rho}=5.63 \times 10^{-3}
\end{array} .\right.
$$

Before putting into use in practice, we should to solve the $\int \frac{d_{S}}{\mu d^{4}}$ in the formula.

\section{B. Integral Calculations in Different Cases}

There are several classic cases when we calculate the integral listed as follows.

1) Case 1: Infinite plate induction surface

Initial condition: the minimum distance between transmitting coil and infinite plate induction is $d$; the dimension of the plate is infinite. 
Object: Numerical or analytic solution of $\int \frac{d_{S}}{\mu d^{4}}$.

Solution:

By arbitrarily selecting an infinitesimal annulus of width $d r$ on plate as in Figure 1, we have

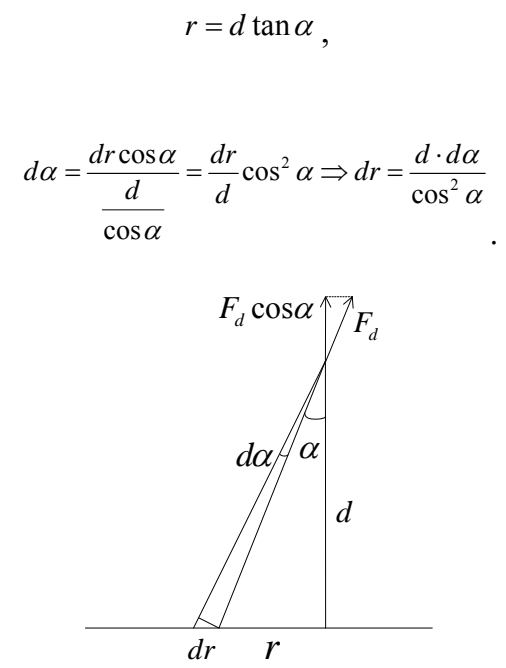

FIGURE I. INFINITE PLATE INDUCTION SURFACE

We get the area of the infinitesimal annular surface

$$
\begin{aligned}
& \boldsymbol{d r s}=2 \pi \boldsymbol{r} \cdot \boldsymbol{d} \boldsymbol{r} \\
& =2 \pi \boldsymbol{d} \tan \alpha \frac{\boldsymbol{d} \cdot \boldsymbol{d} \alpha}{\cos ^{2} \alpha} \\
& =2 \pi \boldsymbol{d}^{2} \frac{\tan \alpha}{\cos ^{2} \alpha} \cdot \boldsymbol{d} \alpha
\end{aligned}
$$

and then

$$
\begin{aligned}
& \int \frac{\boldsymbol{d}_{\boldsymbol{s}}}{\mu \boldsymbol{d}^{4}} \equiv \frac{1}{\mu} \int_{0}^{\alpha \max } \frac{\boldsymbol{d r s} \cdot \cos \alpha}{\left(\frac{\boldsymbol{d}}{\cos \alpha}\right)^{4}} \cdot \cos \alpha \\
& =\frac{1}{\mu} \int_{0}^{\alpha \max } 2 \pi \boldsymbol{d}^{2} \frac{\tan \alpha}{\cos ^{2} \alpha} \cdot \frac{\cos \alpha^{2}}{\left(\frac{\boldsymbol{d}}{\cos \alpha}\right)^{4}} \cdot \boldsymbol{d} \alpha \\
& =\frac{2 \pi}{\mu \boldsymbol{d}^{2}} \int_{0}^{\alpha \max } \tan \alpha \cos ^{4} \alpha \cdot \boldsymbol{d} \alpha \\
& =\frac{2 \pi}{\mu \boldsymbol{d}^{2}} \int_{0}^{\alpha \max } \sin \alpha \cos ^{3} \alpha \cdot \boldsymbol{d} \alpha \\
& =-\frac{2 \pi}{\mu \boldsymbol{d}^{2}} \int_{0}^{\alpha \max } \cos ^{3} \alpha \cdot \boldsymbol{d} \cos \alpha=\left.\frac{2 \pi}{\mu \boldsymbol{d}^{2}} \cdot \frac{1}{4} \cos ^{4} \alpha\right|_{\alpha \max } ^{0} \\
& =\frac{\pi}{2 \mu \boldsymbol{d}^{2}}\left(1-\cos ^{4} \alpha \max \right)
\end{aligned}
$$

For the whole surface of a infinitely large plate, $\alpha \max =\pi / 2$, so we have

$$
\int \frac{d_{S}}{\mu d^{4}} \equiv \frac{\pi}{2 \mu d^{2}}
$$

2) Case 2: Spherical induction surface

Initial condition: the minimum distance between transmitting coil and infinite plate induction is $h$; the radius of the sphere is $R$.

Object: Numerical or analytic solution of $\int \frac{d_{S}}{\mu d^{4}}$.

Solution:

We take an infinitesimal annulus $G G_{2}$ with the center axis $A O$ on spherical induction surface (refer with: Figure 2). Combing the figure, we get these relations:

$$
\begin{gathered}
G E=R \cdot \sin \beta, \\
\frac{O G}{A G}=\frac{\sin \alpha}{\sin \beta} \Rightarrow A G=R \cdot \frac{\sin \beta}{\sin \alpha}, \\
\angle G_{1} G G_{2}=\angle D G F \\
=\angle E G F+\angle E G D \\
=\alpha+\beta
\end{gathered}
$$

$\tan \alpha=\frac{G E}{A E}=\frac{O G \cdot \sin \beta}{h+R-R \cdot \cos \beta}$

$\Rightarrow \alpha=\arctan \frac{R \cdot \sin \beta}{h+R-R \cdot \cos \beta}$,

$$
\beta \max =\arccos \frac{R}{R+h}
$$

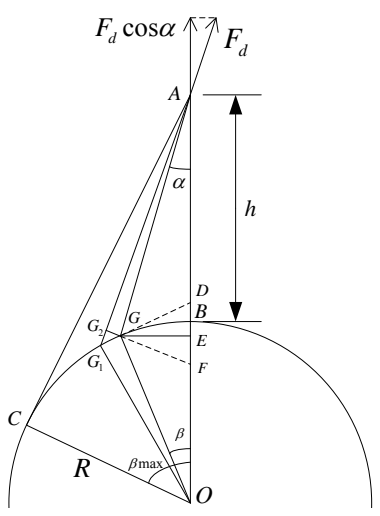

FIGURE II. SPHERICAL INDUCTION SURFACE 
So, we can get

$$
\begin{aligned}
& \int \frac{d_{S}}{\mu d^{4}} \equiv \int_{0}^{\beta \max } \frac{[R \cdot d \beta \cdot \cos (\alpha+\beta)] \cdot(2 \pi R \cdot \sin \beta)}{\mu\left(R \cdot \frac{\sin \beta}{\sin \alpha}\right)^{4}} \cdot \cos \alpha \\
& =\frac{2 \pi}{\mu R^{2}} \int_{0}^{\beta \max } \frac{\cos (\alpha+\beta) \cdot \sin ^{4} \alpha \cdot \cos \alpha}{\sin ^{3} \beta} d \beta
\end{aligned}
$$

When we know the height $h$, spherical radius $R$, and permeability $\mu$, above integral can be solved numerically within Matlab software as follows.

$\%$ solving the integral of spherical induction surface.

$h=120 * 1 e 3 ; \%$ the minimum distance between the coil and the spherical surface is $120 \mathrm{~km}$

$R=6371 * 1 e 3 ; \%$ spherical radius is $6371 \mathrm{~km}$

$\mathrm{mu} 0=4 * p i * 1 e-7 ; \%$ permeability of vacuum/atmosphere

betamax $=\operatorname{acos}(R /(R+h)) ; \%$ the maximum border angle about sphere center

syms beta;\% integration variable

alpha=atan $\left(R^{*} \sin (\right.$ beta $) /\left(h+R-R^{*} \cos (\right.$ beta $\left.\left.)\right)\right) ; \%$ the maximum border angle of the coil

FinalValue $=2 *$ pi $/(m u 0 * R \wedge 2) * \operatorname{int}((\cos ($ alpha + beta $) *(\sin ($ alpha $)) \wedge *$ $\cos ($ alpha $)) /(\sin ($ beta $)) \wedge 3$, beta,0,betamax $) ; \%$ integral expression

$F V=$ eval(FinalValue) $\%$ getting the solution of the integral

3) Case 3: $N$ regular polygon induction surface with sides of a

Initial condition: the minimum distance between transmitting coil and $n$ regular polygon plate induction is $h$; the side dimension of the regular polygon is $a$.

Object: Numerical or analytic solution of $\int \frac{d_{S}}{\mu d^{4}}$.

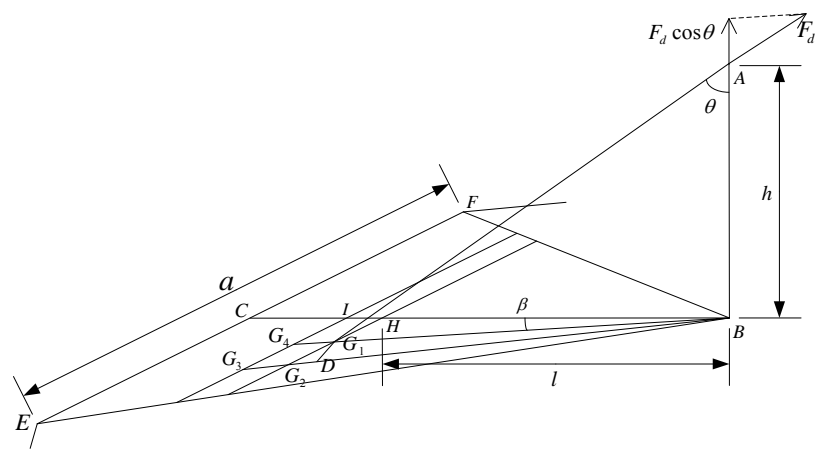

FIGURE III. SPHERICAL $N$ REGULAR POLYGON INDUCTION SURFACE WITH SIDES OF $a$

Solution:

We take the range of $G_{1} G_{2} G_{3} G_{4}$ as an infinitesimal area $S$ on the induction surface. Combing Figure 3, we get these relations as follows:

$$
B G_{1}=\frac{B H}{\cos \beta}=\frac{l}{\cos \beta},
$$

$$
G_{1} D \perp G_{2} D \Rightarrow \angle G_{2} G_{1} D=\beta
$$

$$
G_{1} D \approx B G_{1} \cdot d \beta
$$

Combing (13), (14), and (15) products

$$
\begin{gathered}
G_{1} G_{2}=\frac{G_{1} D}{\cos \angle G_{2} G_{1} D}=\frac{B G_{1} \cdot d \beta}{\cos \beta}=\frac{\frac{l}{\cos \beta} \cdot d \beta}{\cos \beta}=\frac{l}{\cos ^{2} \beta} d \beta, \\
S \approx G_{1} G_{2} \cdot H I=\frac{l}{\cos ^{2} \beta} d \beta \cdot d l \\
\theta=\arctan \frac{B G_{1}}{h}=\arctan \frac{\frac{l}{\cos \beta}}{h}=\arctan \frac{l}{h \cdot \cos \beta},
\end{gathered}
$$

and then

$$
\begin{gathered}
d=A G_{1}=\frac{h}{\cos \theta}=\sqrt{h^{2}+\left(\frac{l}{\cos \beta}\right)^{2}}, \\
\beta \max =\angle C B E=\frac{1}{2} \cdot \frac{2 \pi}{n}=\frac{\pi}{n}, \\
l \max =C B=\frac{\frac{a}{2}}{\tan \angle C B E}=\frac{a}{2 \tan \frac{\pi}{n}} .
\end{gathered}
$$

With above equations, the integral can be further deal with as follows:

$$
\begin{aligned}
& \int \frac{d_{S}}{\mu d^{4}} \equiv 2 n \int_{0}^{l \max } \int_{0}^{\beta \max } \frac{S \cdot \cos \theta}{\mu d^{4}} \cdot \cos \theta \\
& =2 n \int_{0}^{l \max } \int_{0}^{\beta \max } \frac{\frac{l \cdot \cos ^{2} \theta}{\cos ^{2} \beta} \cdot d \beta \cdot d l}{\mu\left[h^{2}+\left(\frac{l}{\cos \beta}\right)^{2}\right]^{2}}
\end{aligned}
$$




$$
\begin{aligned}
& =2 n \int_{0}^{l \max } \int_{0}^{\beta \max } \frac{l \cdot \frac{h^{2}}{h^{2}+\left(\frac{l}{\cos \beta}\right)^{2}}}{\mu\left[h^{2}+\left(\frac{l}{\cos \beta}\right)^{2}\right]^{2} \cdot \cos ^{2} \beta} \cdot d \beta \cdot d l \\
& =2 n \int_{0}^{l \max } \int_{0}^{\beta \max } \frac{l \cdot h^{2}}{\mu\left[h^{2}+\left(\frac{l}{\cos \beta}\right)^{2}\right]^{3} \cdot \cos ^{2} \beta} \cdot d \beta \cdot d l \\
& =\frac{2 n h^{2}}{\mu} \int_{0}^{l \max } \int_{0}^{\beta \max } \frac{l}{\left[h^{2}+\left(\frac{l}{\cos \beta}\right)^{2}\right]^{3} \cdot \cos ^{2} \beta} \cdot d \beta \cdot d l .
\end{aligned}
$$

When we know the $n$ regular polygon sides' dimension $a$, height $h$, and permeability $\mu$, the integral can be solved numerically within Matlab software as follows.

$\%$ solving the integral of $n$ regular polygon induction surface. surface

$h=9.95 e-3 ; \%$ the minimum distance between the coil and the polygon

$n=4 ; \%$ the case of a square

$a=0.2 ; \%$ square edge length $200 \mathrm{~mm}$

mu $0=4 * p i * 1 e-7 ; \%$ permeability of vacuum/atmosphere

betamax $=p i / n ; \%$ the maximum border angle

$\operatorname{lmax}=a /(2 * \tan (\mathrm{pi} / \mathrm{n})) ; \%$ the maximum border length

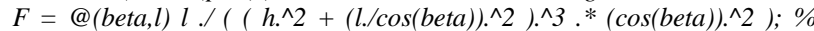
integral expression

$Q=$ dblquad(F,0,betamax,0,lmax); \% solving the integral

eff $=2 * n * h \wedge 2 / m u 0 ; \%$ the common coefficient

$F V=e f f * Q \%$ final numerical solution of aim integral

Integral $=p i /(2 * m u 0 * h \wedge 2) \%$ contrasting the solution with the infinitely large plate

4) Case 4: Rectangle induction surface with sides of $a \times b$

Initial condition: the minimum distance between transmitting coil and rectangle induction is $h$; the side dimensions of the rectangle are $a \times b$.

Object: Numerical or analytic solution of $\int \frac{d_{S}}{\mu d^{4}}$.

The solution method is as similar as Case 3, and the final solution we got is shown as follows

$$
\begin{aligned}
& \int \frac{d_{S}}{\mu d^{4}} \equiv \frac{4 h^{2}}{\mu}\left(\int_{0}^{a / 2} \int_{0}^{\arctan (b / a)} \frac{l}{\left[h^{2}+\left(\frac{l}{\cos \beta}\right)^{2}\right]^{3} \cdot \cos ^{2} \beta} \cdot d \beta \cdot d l\right. \\
& \left.+\int_{0}^{b / 2} \int_{0}^{\arctan (a / b)} \frac{l}{\left[h^{2}+\left(\frac{l}{\cos \beta}\right)^{2}\right]^{3} \cdot \cos ^{2} \beta} \cdot d \beta \cdot d l\right)
\end{aligned}
$$

Numerical calculation programme code is as follows.

$\%$ solving the rectangle induction surface

$h=9.95 e-3 ; \%$ the minimum distance between the coil and the rectangle surface $(m)$.

$a=0.2 ; \%$ rectangle side length

$b=0.2 ; \%$ rectangle side length

$\mathrm{mu} 0=4 *$ pi*1e-7;\% permeability of vacuum/atmosphere

$F 1=@($ beta,l) l./ ( ( h.^2 + (l./cos(beta)).^2 ).^3 .* $(\cos ($ beta $)) . \wedge 2) ; \%$ integral expression

Q1 = dblquad(F1,0,atan(b/a),0,a/2); \% solving the integral

$F 2=@($ beta,l) l./ ( ( h. $12+(l . / \cos ($ beta $)) . \wedge 2) . \wedge 3 . *(\cos ($ beta $)) . \wedge 2) ; \%$ integral expression
$Q 2=\operatorname{dblquad}(F 2,0, \operatorname{atan}(a / b), 0, b / 2) ; \%$ solving the integral

eff $=4 * h \wedge 2 / m u 0 ; \%$ the common coefficient

$F V=e f f^{*}(Q 1+Q 2) \%$ final numerical solution of aim integral

When the height $h$ is very small, for more approaching to the ideal value, we set $h=\sqrt{h_{\min } \cdot h_{\max }}$, where $h_{\min }$ is the minimum height of the coil, and $h_{\max }$ is the maximum height.

\section{Experimental Proof}

Lastly, we've verified its validity in practical testing (refer with: Table 1). Figure 4 shows our tested model. When we supply the coil with a adjustable AC power, we can get loss weight data as in Table 1. From the coil parameter and measuring data, we can check out the the experimental result is well according to above formula calculation result (refer with: Equation 17). When we change some different coils and metal plates [4], it is also verified correct [5] in all of the other cases $[6]$.

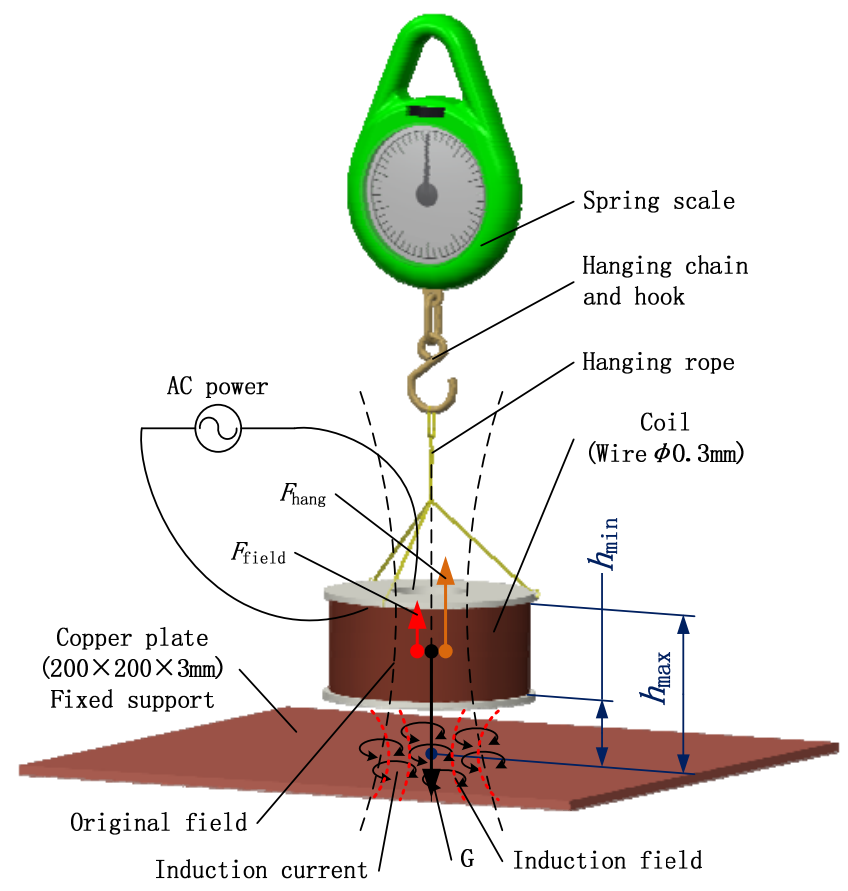

FIGURE IV. TESTED MODEL AND ITS FIELD DISTRIBUTIONS

TABLE I. SUPPLY MAXIMUM FREQUENCY 400HZ, MAXIMUM AC CURRENT 0.668A, CIRCUIT RESISTANCE $144 \Omega$

\begin{tabular}{|c|c|c|c|c|c|}
\hline Frequency (Hz) & 400 & 200 & 100 & 60 & 45 \\
\hline On-load AC voltage (V) & 299.5 & 176.5 & 135.4 & 115 & 116.5 \\
\hline Output power (W) & 46 & 68 & 74.9 & 70.2 & 73.7 \\
\hline Mass indication (g) & 25 & 50 & 70 & 90 & 100 \\
\hline Loss Mass (g) & 95 & 70 & 50 & 30 & 20 \\
\hline Loss weight (N) & 0.931 & 0.686 & 0.49 & 0.294 & 0.196 \\
\hline
\end{tabular}

\section{Summary}

By far, we've solved the AC current-carrying coil problem completely. Though it is solved and measured coefficients in low-frequency case, that's also considerate satisfied to highfrequency coil [7] in theory. It provides us a newer thinking to 
interpret the electromagnetic wave thrust problem and can help us to understand the hot area of electric-magnetic drive system more deeply.

\section{ACKNOWLEDGMENT}

This research was financially supported by Electromagnetic Propulsion Power Systems, the Important Deployed Project of the Thirteenth Five-year Plan of Hefei Institutes of Physical Science of Chinese Academy of Science; and Calculation for Optimum Design for High Homogeneous MRI Superconducting-magnets and its Relevant Software Development, Anhui Provincial Natural Science Foundation.

\section{REFERENCES}

[1] Kamm Jochen, Becken Michael, and Pedersen Laust, "Inversion of slingram electromagnetic induction data using a Born approximation," Geophysics, v 78, n 4, p E201-E212, June 01, 2013.

[2] Yin Wuliang, Peyton A.J., and Dickinson Stephen J., "Simultaneous measurement of distance and thickness of a thin metal plate with an electromagnetic sensor using a simplified model," IEEE Transactions on Instrumentation and Measurement, v 53, n 4, p 1335-1338, August 2004.

[3] Zhang YAN, Xiang-yu CHENG, and Wen-ge CHEN, "Theoretical Modeling for a Kind of Electric-magnetic Drive System," unpublished.

[4] Lee K.S., Kim, S.W., and Eom, D.H., "Temperature distribution and bending behaviour of thick metal plate by high frequency induction heating," Materials Research Innovations, v 15, n SUPPL. 1, p S283S287, February 2011.

[5] Fang Fang, Wang Tiejun, and Li Bing, "Analysis and design of electromagnetic vehicles climbing on steel plates," 2015 IEEE International Conference on Mechatronics and Automation, ICMA 2015 , p 1223-1227, September 2, 2015.

[6] Asten Michael W., Duncan, and Andrew C., "Fast approximate EM induction modeling of metallic and UXO targets using a permeable prism," Journal of Applied Geophysics, v 61, n 3-4, p 235-242, March 19, 2007.

[7] He Peng, and Wang Hong-Qin, "Numerical simulation of heating process for copper pipe-plate high frequency induction brazing and its application," Journal of Harbin Institute of Technology, v 37, n SUPPL. 1, p 95-98, May 2005. 